\title{
Linear Ultrasonic Array incorporating a Cantor Set Fractal Element Configuration
}

\author{
H. Fang, Z. Qiu, R. L. O'Leary and A. Gachagan \\ Centre for Ultrasonic Engineering \\ University of Strathclyde \\ Glasgow, UK G1 1XW \\ Email: haoyu.fang@strath.ac.uk
}

\author{
A. J. Mulholland \\ Department of Mathematics \\ University of Strathclyde \\ Glasgow, UK G1 1XH
}

\begin{abstract}
The resonance frequency of an active element in a piezoelectric ultrasonic transducer is dependent on its length scale. Inspired by natural occurring auditory systems, incorporation of elements with varying length scales in the piezoelectric transducer design can result in a wider operational bandwidth. In this paper, a mathematical algorithm was developed first to define the feature of a fractal geometry called the Cantor Set (CS), then a series of parameter sweep simulations are performed to design a CS fractal array transducer and a conventional array transducer and optimize their performance. The behaviors of these two array transducers were explored theoretically, using finite element modeling and experimentally using the scanning laser vibrometry. The FE simulation results and experimental results correlate well with each other, which indicates an approximate $30 \%$ operating bandwidth enhancement and a $5 \mathrm{~dB}$ side lobe reduction can be achieved by the $\mathrm{CS}$ fractal array compared to the conventional linear array design.
\end{abstract}

Keywords-Fractal Ultrasonic Array; Array Characterization; Wide Bandwidth;

\section{INTRODUCTION}

A typical ultrasonic array refers to an ultrasound transducer design comprising a number of closely spaced elements, where the centre-to-centre spacing is typically less than $\lambda 12$ [1]. Such arrays are employed to allow electronic beam steering within the insonified medium [2]. Consequently, the ultrasound array technique is extensively used in clinical diagnostic and therapeutic applications such as real-time blood flow measurement [3] and non-invasive surgery [4] and also in industrial applications such as nondestructive testing and evaluation [5].

The performance of an ultrasound array system can be tailored to the application by various schemes [6]:

a) Electronic tuning,

b) Optimising the array microstructure,

c) Design of the active and passive material.

As an example of using an electrical tuning method, Rodriguez et al. designed a low cost L-C matching network to eliminate the energy loss caused by the electrical impedance mismatch between the ultrasonic device and the driving equipment, improving the signal sensitivity of the ultrasonic device [7]. In terms of improving the array performance by considering the active piezoelectric material, Wong et al. proposed a high-frequency phased array transducer design with a PMN-PT single crystal material, which exhibited a higher operational bandwidth compared with other piezoceramic designs [8]. Moreover, enhanced array performance can be achieved through modification of the active layer structure. Yang et al. reported that the cross talk between two adjacent elements of an ultrasonic array can be reduced by using a 1-3 piezocomposite design with a pseudo-random pillar configuration rather than a conventional periodic structure [9].

In the authors' previous work, a single element piezoelectric composite transducer was developed using the Sierpinski Gasket (SG) fractal geometry [10]. This single element SG fractal composite transducer exhibited a larger bandwidth in both transmission and reception mode compared to an equivalent conventional parallelepiped 1-3 composite design. However, due to the fabrication limitation, it is difficult to extend this single element SG fractal composite design to an array transducer. Subsequently, in this paper, another fractal geometry known as the Cantor Set (CS), shown in the Fig. 1, will be used to design and manufacture a 2-2 fractal composite and an array transducer with 24 elements will be produced using this 2-2 CS fractal composite plate. Compared with the SG fractal composite previously reported, this CS fractal composite can be manufactured easily using the conventional dice-and-fill technique [11]. The performance of the CS fractal array will be compared to an equivalent conventional array design with a regular periodic 2-2 composite structure.

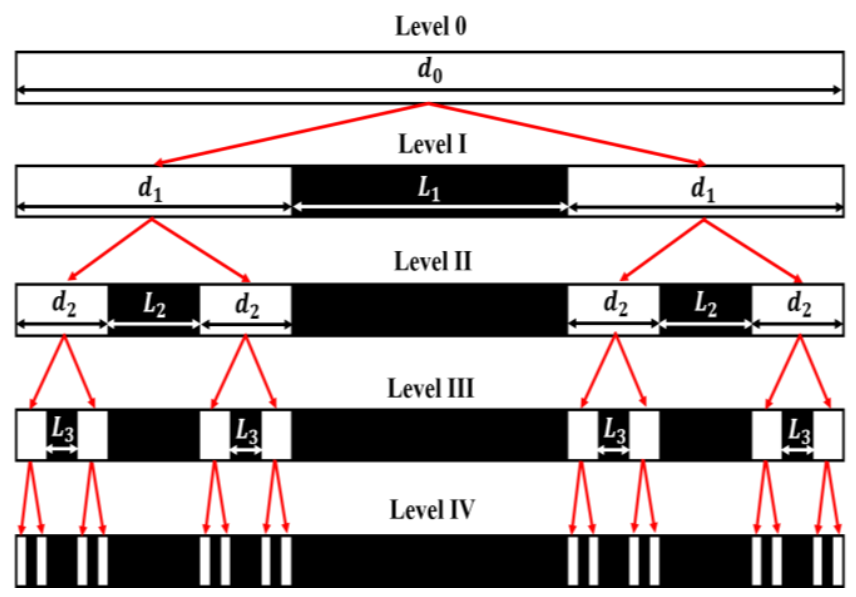

Fig. 1: Schematic diagram of the first five fractal generation levels of the CS fractal geometry (Black: Ceramic Pillar; White: Polymer Filler).

The core funding for Mr Fang's studentship has been provided by the University of Strathclyde and the work has been carried out under the auspices of the UK Research Centre for NDE (EPSRC Grant EP/L022125/1). 


\section{Finite ELEMENT ModelLing}

\section{A. CS Fractal Geometry Generation Algorithm}

An iteration rule for defining the features of the CS fractal geometry was developed in terms of a scaling factor, $k$ (where $0<k<0.5$ ). As shown in Fig. 1, a CS fractal geometry of total length $d_{0}$ at generation Level 0 is iterated to the next generation level with a scaling factor, $k$. The geometrical feature of this CS fractal geometry at generation Level $\mathrm{N}$ can be determined by the value of $d_{n}$ and $L_{n}$ using the following equations,

$$
\begin{gathered}
d_{n}=d_{0} \cdot k^{n} \\
L_{n}=d_{0} \cdot k^{n-1} \cdot(1-2 k),(n \geq 1)
\end{gathered}
$$

\section{B. Array Single Element Design Evaluation}

In order to explore the influence of adopting this CS fractal geometry as the structure of an array transducer design, two FE models (PZFlex, Thornton Tomasetti) representing a single array element incorporating a generation Level II CS fractal device or a conventional periodic 2-2 piezocomposite microstructure were built, as illustrated in Figs. 2(a) and (b) respectively. The thickness of each array design is $1.5 \mathrm{~mm}$, corresponding to a mechanical resonance frequency approximately $1 \mathrm{MHz}$. PZT5H ceramic and CY1300/HY1301 polymer are chosen to be the active phase and passive phase materials respectively. In order to optimise the bandwidth performance of these two models, a number of parameter sweep simulations were performed. The resulting Q-factors, defined as the ratio of centre frequency to the half amplitude bandwidth in the conductance spectrum, were calculated to describe the device bandwidth. A low value of the Q-factor means a broadband device can be realised. (a)

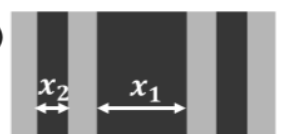

(b)

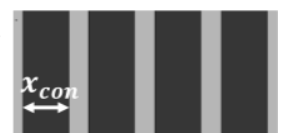

Fig. 2: Schematic diagram of a single element within the array, (a) Level II CS fractal; (b) 2-2 piezocomposite (Black: Ceramic; Gray: Polymer)

As illustrated in Fig. 2, the first parameter sweep simulation was performed by keeping the scaling factor, $k$, of one CS fractal array element to be 0.32 , where a standard CS fractal geometry at generation Level II can be obtained, then varying the value of the pillar width at the second generation level, $x_{2}$, from $0.1 \mathrm{~mm}$ to $1.0 \mathrm{~mm}$ with a step size of $0.05 \mathrm{~mm}$. The resulting conductance spectrum and Q-factor for different values of $x_{2}$ are shown in Fig. 3 and Fig. 4, where the peak of each main lobe marked with a red dot.

From Fig. 3 and Fig. 4, it can be identified that the lowest Q-factor value calculated from the main lobe of the conductance spectra can be achieved when $x_{2}$ is $0.15 \mathrm{~mm}$, which means the highest bandwidth was obtained compared to other conditions.

After determining a desired value of $x_{2}$, the second parameter sweep simulation was performed by fixing the value of $x_{2}$ to be $0.15 \mathrm{~mm}$ and varying the scaling factor, $k$, from 0.2 to 0.4 with a step of 0.01 . The resulting conductance and Q-factor for each different scaling factor, $k$, are shown in Fig. 5 and Fig. 6.

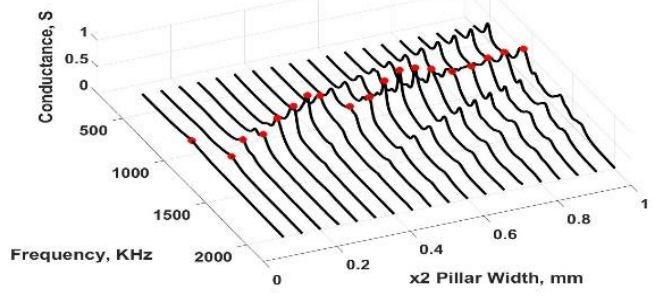

Fig. 3: FE derived conductance spectrum for different values of $x_{2}$ in the CS fractal array design

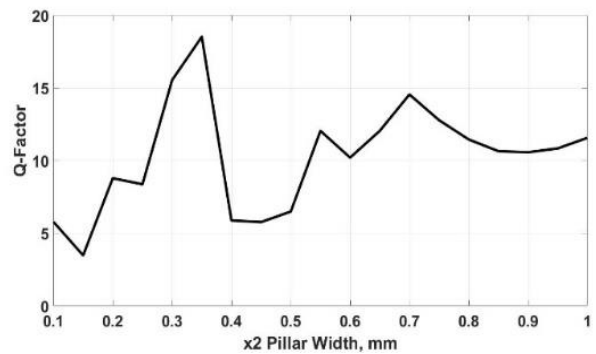

Fig. 4: Q-factor for different values of $x_{2}$ in the CS fractal array design

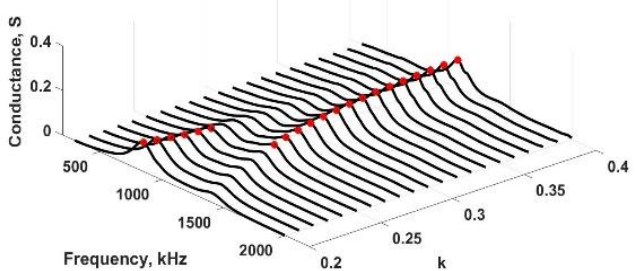

Fig. 5: FE derived conductance spectrum for different values of $k$ in the CS fractal array design

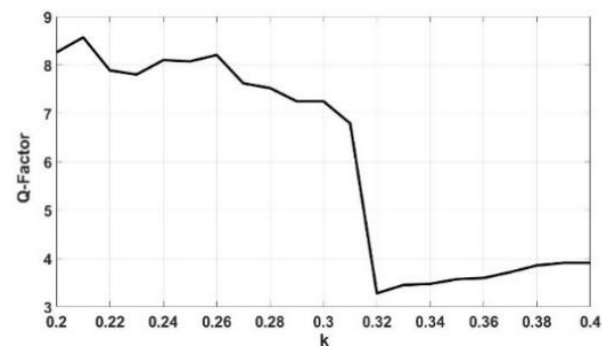

Fig. 6: Q-factor for different value of $\mathrm{k}$ in the CS fractal array design

In Fig. 5 and Fig. 6, it is shown that when $k$ equals 0.32 , the lowest Q-factor calculated from the conductance main lobe can be obtained, where the highest bandwidth can be achieved.

The last parameter sweep simulation determines an optimal design for the conventional 2-2 piezocomposite array element design. By setting the value of the pillar width of one conventional array element, $x_{\text {con }}$, as shown in Fig. 2, to be 
$0.15 \mathrm{~mm}$, which is the same as $x_{2}$ in the CS fractal design, then varying the saw width from $0.1 \mathrm{~mm}$ to $0.3 \mathrm{~mm}$, with a step size of $0.05 \mathrm{~mm}$, the resulting predicted conductance spectrum and the Q-factor are shown in Fig. 7 and Fig. 8.

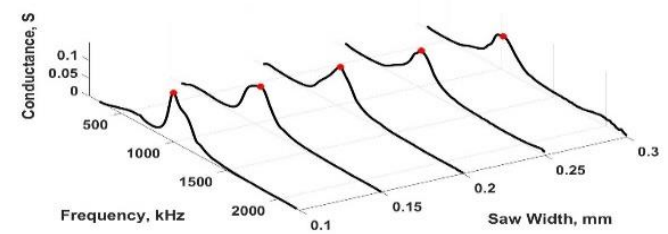

Fig. 7: FE derived conductance spectrum for different values of the saw width in the conventional array design

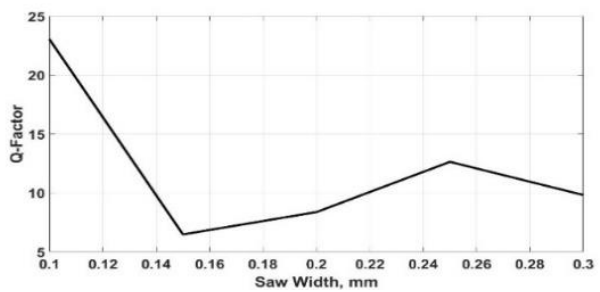

Fig. 8: Q-factor for different values of the saw width in the conventional array design

As illustrated in Figs.7 and 8, the highest bandwidth for a conventional array element can be achieved when the saw width equals $0.15 \mathrm{~mm}$.

The parameter sweep simulations have produced optimized 2-2 piezocomposite microstructures designed to maximise bandwidth for both the CS fractal and the conventional periodic array element configurations. For the CS fractal array design, the scaling factor, $k$, and the pillar width at the second generation level, $x_{2}$, were set to be 0.32 and $0.15 \mathrm{~mm}$ respectively, where the pillar width at the first generation level and saw width is be calculated to be $0.47 \mathrm{~mm}$ and $0.145 \mathrm{~mm}$ respectively. For the conventional array design, the pillar width, $x_{\text {con }}$, and the saw width were both determined as $0.15 \mathrm{~mm}$. Both designs have a similar ceramic volume fraction, which is $58.5 \%$ for the CS fractal array design and $50.0 \%$ for the conventional design.

\section{Single Element Transmitting Voltage Response Simulation}

The FE models simulated one element of both the CS fractal and the conventional array operating in a water load and the simulated TVR is shown in Fig. 9.

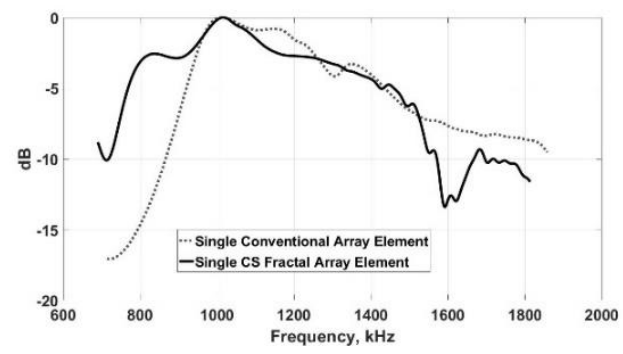

Fig. 9: TVR Spectrum of a single element of the CS fractal and the conventional array
From Fig. 9, it can be seen that the predicted $-6 \mathrm{~dB}$ bandwidth of the CS fractal array element and the conventional array element is $71.7 \%$ and $55.6 \%$ respectively, where a $28.9 \%$ bandwidth improvement can be achieved by a single CS fractal array element with respect to a single conventional array element.

\section{Array Beam Profile Evaluation}

Based on the selected parameters from Section II. B, FE models of a 24-element CS fractal array and a 24-element conventional array were built. Fig. 10 (a) and (b) display the simulated beam profiles of the CS fractal array transducer and the conventional array transducer, when both devices were focused at $15 \mathrm{~mm}$ away from the front face of the device in the water load. It can be determined that the side lobe of the $\mathrm{CS}$ fractal array is $-5 \mathrm{~dB}$ lower than the conventional array. (a)

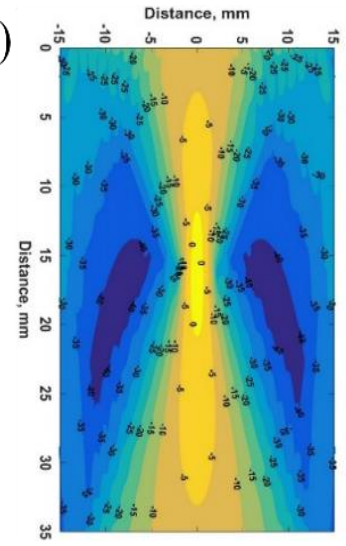

(b)

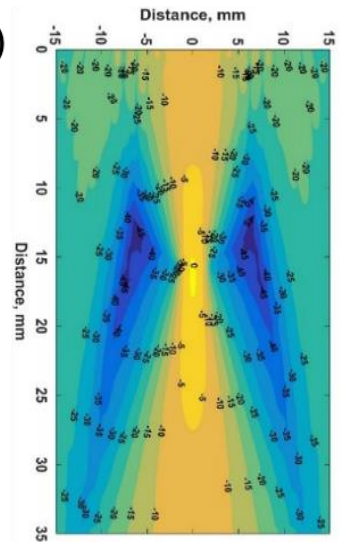

Fig. 10: Simulated beam profile, (a) CS fractal array (b): Conventional Array (Focused at $15 \mathrm{~mm}$ )

\section{EXPERIMENTAL VALIDATION}

\section{A. CS Fractal Array Substrate Manufacturing}

The 2-2 piezocomposite active layers for both the CS fractal array and the conventional array design were fabricated using the traditional dice-and-fill technique. However, because there are two different sizes of pillars in the CS fractal array composite active layer, three groups of cuts, represented by three different colours, as shown in Fig. 11, were needed to fabricate this CS fractal composite substrate plate, with all three groups of cuts sharing the same pitch value.

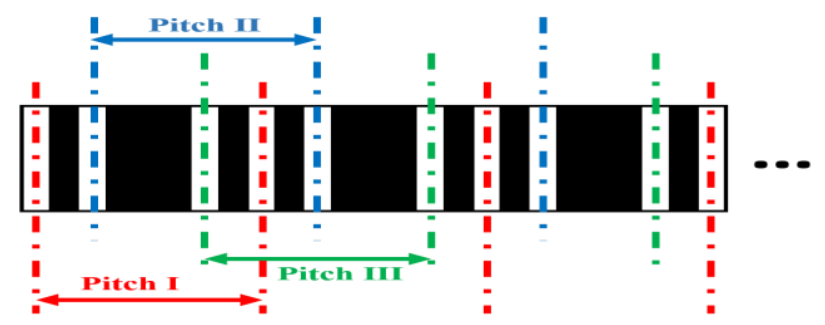

Fig. 11: Schematic of fabricating CS fractal array composite active layer

\section{B. Impedance Profile}

The measured electrical impedance profiles for the two array microstructures (one for the CS fractal and one for the conventional array) are as shown in Fig. 12. 


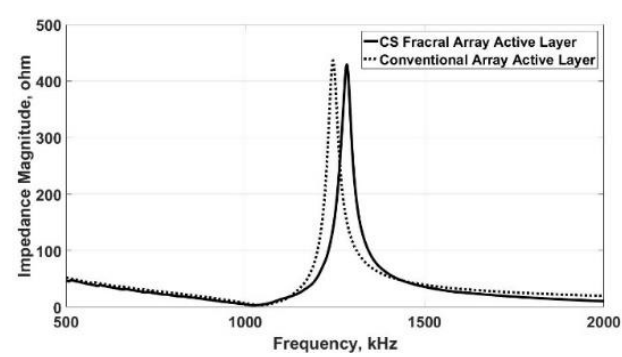

Fig. 12: Measured electrical impedance magnitude spectrum of the CS array and conventional array composite active layer

It can be seen from Fig. 12 that the mechanical resonance frequency of the CS fractal array and conventional array active layer are located at $1022 \mathrm{kHz}$ and $1047 \mathrm{kHz}$ respectively. The electromechanical coupling coefficient, $k_{t}$, is 0.62 for the CS fractal array active layer and 0.56 for the conventional array active layer, and so a better energy conversion and improved bandwidth can be realised by the CS fractal design.

\section{Displacement Profile}

The manufactured two active layers has the ability of acting as two 24-element array transducers respectively, however at this stage, they were driven as whole respectively with common electrodes. The surface vibration behaviours of the two array active layers were evaluated using Huygens model and measured data using a Polytec PSV-300 scanning laser vibrometer (LDV) (Polytec Inc.). The resulting surface average velocity frequency response and beam profile for both devices are shown in Fig. 13 and Fig. 14(a) and (b), respectively.

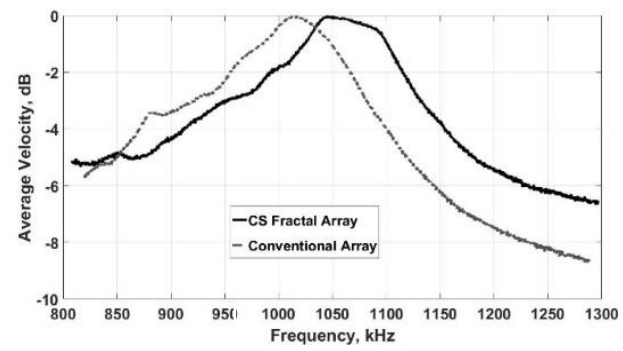

Fig. 13: Measured average surface velocity for the CS fractal and the conventional array as a function of the frequency

From Fig. 13, the $-6 \mathrm{~dB}$ bandwidth of the average surface velocity frequency response is $40.2 \%$ for the CS fractal array and $31.9 \%$ for the conventional design. So there is a $32 \%$ bandwidth improvement achieved by the CS fractal array with respect to the conventional array. From Fig. 14, a 4 dB side lobe reduction can be achieved by the CS fractal array design compared to the conventional array design.

\section{CONCLUSION}

This paper describes the implementation of the CS fractal geometry as the structure of a linear array design for the purpose of improving the performance of an ultrasonic array transducer. A mathematical algorithm was developed to define the geometrical features of the CS fractal geometry and a series of parameter sweep simulations optimized the design of the CS fractal array. The FE simulation results correlated well with the experimental results, where an approximately $30 \%$ bandwidth improvement and $5 \mathrm{~dB}$ side lobe reduction were achieved by the CS fractal array design compared to an equivalent conventional array design. (a)

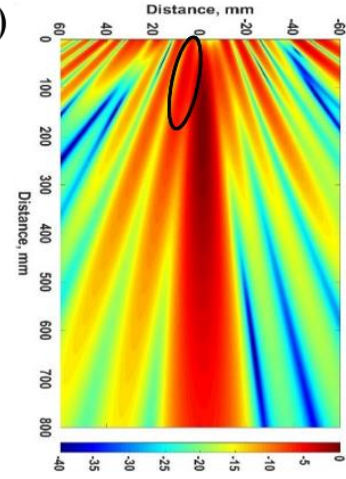

(b)

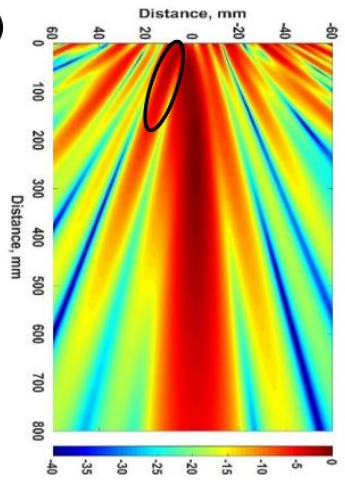

Fig. 14: Measured active layer beam profile at their nature focus, (a) CS fractal array; (b) conventional array

\section{REFERENCES}

[1] B. W. Drinkwater and P. D. Wilcox, "Ultrasonic arrays for non destructive evaluation: A review," NDT E Int., vol. 39, no. 7, pp. 525541, 2006.

[2] P. R. Smith, D. M. J. Cowell, B. Raiton, C. V. Ky, and S. Freear, "Ultrasound array transmitter architecture with high timing resolution using embedded phase-locked loops," IEEE Trans. Ultrason. Ferroelectr. Freq. Control, vol. 59, no. 1, pp. 40-49, 2012.

[3] L. Zhang, X. Xu, C. Hu, L. Sun, J. T. Yen, J. M. Cannata, and K. K. Shung, "A high-frequency, high frame rate duplex ultrasound linear array imaging system for small animal imaging," Ultrason. Ferroelectr. Freq. Control. IEEE Trans., vol. 57, no. 7, pp. 1548-1557, 2010.

[4] E. B. Hutchinson, "Intracavitary ultrasound phased arrays for noninvasive prostate surgery," IEEE Trans. Ultrason. Ferroelectr. Freq. Control, vol. 43, no. 6, pp. 1032-1042, 1996.

[5] S. Mahaut, O. Roy, C. Beroni, and B. Rotter, "Development of phased array techniques to improve characterisation of defect located in a component of complex geometry," Ultrasonics, vol. 40, no. 1-8, pp. $165-169,2002$

[6] Q. Zhou, K. Ho, H. Zheng, W. Qiu, and K. K. Shung, "Piezoelectric single crystal ultrasonic transducers for biomedical applications," J. Prog. Mater. Sci., vol. 66, pp. 87-111, 2014.

[7] M. Garcia-Rodriguez, J. Garcia-Alvarez, Y. Yañez, M. J. GarciaHernandez, J. Salazar, A. Turo, and J. A. Chavez, "Low cost matching network for ultrasonic transducers," Phys. Procedia, vol. 3, no. 1, pp. 1025-1031, 2010.

[8] C. M. Wong, Y. Chen, H. Luo, J. Dai, K. H. Lam, and H. L. wa Chan, "Development of a 20-MHz wide-bandwidth PMN-PT single crystal phased-array ultrasound transducer,"Ultrasonics, vol. 73, pp. 181-186, 2017

[9] H. C. Yang, J. Cannata, J. Williams, and K. K. Shung, "A study of 1-3 pseudo-random pillar piezocomposites for ultrasound transducers," IEEE Int. Ultrason. Symp. IUS, no. c, pp. 1743-1746, 2011

[10] H. Fang, Z. Qiu, R. L. O’Leary, A. Gachagan, and A. J. Mulholland, "Improving the operational bandwidth of a 1-3 piezoelectric composite transducer using Sierpinski Gasket fractal geometry," IEEE Int. Ultrason. Symp. IUS, vol. 2016-Novem, 2016.

[11] H. P. Savakus, K. A. Klicker, and R. E. Newnham, "PZT-epoxy piezoelectric transducers: A simplified fabrication procedure," Mater. Res. Bull., vol. 16, no. 6, pp. 677-680, Jun. 1981. 